CSORDÁS, Hédi Virág

\title{
VISUAL ARGUMENTS: MOVING VS. STILL IMAGES IN WWF CAMPAIGNS ${ }^{1}$
}

\section{Introduction}

Our information age is strongly characterised by mediation, which is closely related to the expansion of visual experience. In the twentieth century, scientists attempted to categorise our media areas. In his widely known work Orality and Literacy: The Technologizing of the Word leading social scientist Walter Ong introduced the terms primary orality, literacy and secondary orality. Primary orality refers to thought and its verbal expression within cultures "totally untouched by any knowledge of writing or print." ${ }^{2}$ Within the concept of literacy he sees communication gradually developing from an oral stage into a stage of print. Secondary orality is dependent on literate culture and the existence of writing, as in the case of a television or radio anchor reading the news. Moreover, as John Walter points out, Ong introduced one further category in a 1996 interview in Composition FORUM: "Ong also uses the term 'secondary visualism' on a few occasions in his unpublished writings to emphasize the increased use of non-textual visual and interactive elements." ${ }^{3}$ This new form of communication is primarily based on pictures - as further evidenced by esteemed philosopher W. J. T. Mitchell's 'pictorial turn' concept ${ }^{4}$. It is thus maintained that a wide range of visual elements, such as pictures, icons, billboards, city lights, short videos and advertisements, significantly influence our thoughts about the world, and can sometimes change our behaviour and attitudes.

\section{Hypothesis}

In this paper I will focus on the visual elements of understanding as they appear in World Wildlife Fund (WWF) public service campaigns.

I will argue that the visual argumentation structures characteristic of pictures can influence the way images are processed. I will also claim that the degree of impact depends on the type of images: whether they are moving or still pictures. In order to substantiate my hypothesis, I will start by introducing WWF and their mission and describing the characteristics of public service campaigns. I will give definitions for some fundamental expressions (systematic and heuristic processing, visual elements, visual arguments,

\footnotetext{
${ }^{1}$ This research is carried ouit in the framework of Integral Argumentation Studies (OTKA - K-109456) of the Doctoral School of Philosophy and History of Science, Budapest University of Technology and Economics.

${ }^{2}$ Walter J. Ong. Orality and Literacy, p. 11.

${ }^{3}$ Kleine, Michael, and Fredric G. Gale, "The Elusive Presence of the Word: An Interview with Walter Ong." Composition FORUM 7.2 (1996): pp. 65-86.http://johnwalter.blogspot.hu/2006/07/ong-on-secondary-oralityand-secondary.html, last accessed 02. 12. 2015.; Notes from Walter J. Ong's Archive

${ }^{4}$ Mitchell, W.J. T., "The pictorial turn”. In: Mitchell, W.J. T. (1994.) Picture Theory
} 
argumentative schemes and so on) which I will repeatedly use in my work. I will then draw up the main components of visual argumentation which are linked to the processing of images. Finally, I will make a comparison between still and moving images, focusing on their dissimilarities. My aim is to demonstrate that there is a connection between argumentation structures and the processing of images (still and moving).

\section{Introducing WWF}

WWF's mission is to build a future in which people live in harmony with nature. They focus on two goals: protecting biodiversity, and reducing humanity's footprint on the natural world. Not only do they identify problems, but they also find solutions, concentrating on achievable targets, policies and results.

This international organization operates in more than 100 countries on 6 continents and employs more than 5000 people worldwide. Their total revenues and incomes are 657 million euros, most of which comes from 5 million supporters. ${ }^{5}$ When we compare the two pie charts below, we can see that the largest segment of WWF's income is donated by individuals (55\%), while on the expenditure side the rates of investment (in awareness and education) are much lower ( $11 \%$ and $3 \%$, respectively). This may cause us to wonder how they can reach their target group and remain economically efficient while their budget is low. As I will point out, it depends on creativity, and on the structures of their visual arguments.

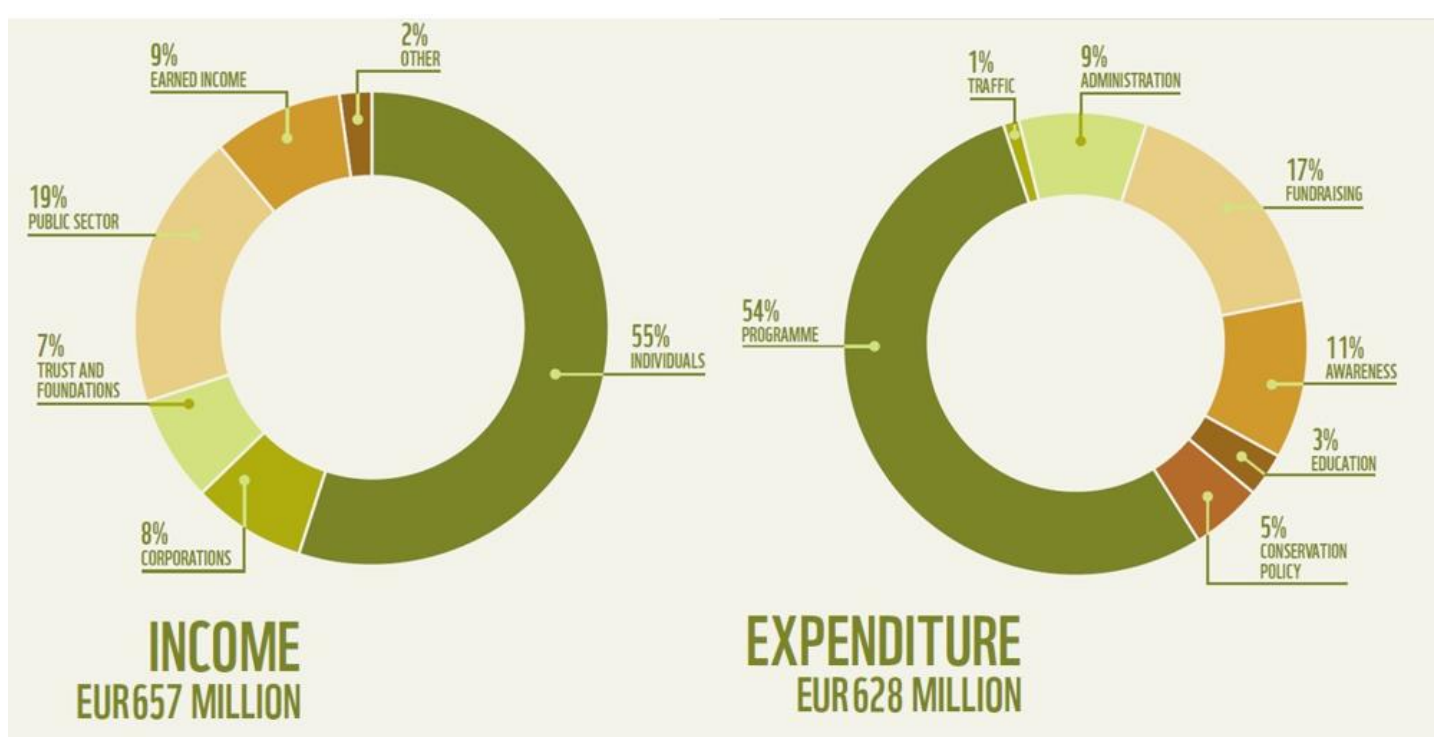

Figure 1. WWF incomes and expenditures in $2014 .^{6}$

\footnotetext{
${ }^{5}$ WWF-US Annual Report 2014. p.

56.http://assets.worldwildlife.org/financial_reports/21/reports/original/2014_Annual_Report.pdf?1418325091 last accessed 11. 12. 2015.

${ }^{6}$ WWF Annual Review 2014. p. 37. http://europe.nxtbook.com/nxteu/wwfintl/annualreview2014/\#/Olast accessed 12. 12. 2015.
} 
My analysis will involve campaigns mounted by local offices. I will go through the short movie It all comes back to you, and some still images, such as If the trees/ice fall, we all fall ${ }^{7}$ and Stop one, stop them all ${ }^{8}$. I will analyse posters against killing animals in order to produce material goods, Bycie pamiqtkq boli ${ }^{9}$ [Being a trophy hurts].

\section{Environmental advertising and marketing communication}

The main message of a public service campaign is usually obvious, but it can be perceived in different ways. The International Chamber of Commerce (ICC) commissions, which are "specialized working bodies composed of business experts who examine major issues of interest to the business world" and establish "rules and codes to facilitate international business transactions"10 have compiled the Framework for Responsible Environmental Marketing Communications, which has the following to say about green claims: "All marketing communications should be judged by their likely impact on the reasonable consumer, having regard to the characteristics of the targeted group and the medium used. A consumer's interpretation of a green claim is affected by the context in which it is presented, the level of knowledge and experience (e.g., professional or sophisticated users versus typical consumers), and [the] form in which it is conveyed."11

I will argue that WWF campaigns follow this advice fairly
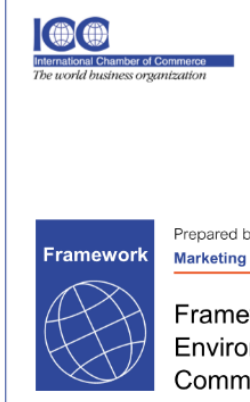

Prepared by ICC Commission on Marketing and Advertising

Framework for Responsible Environmental Marketing Communications

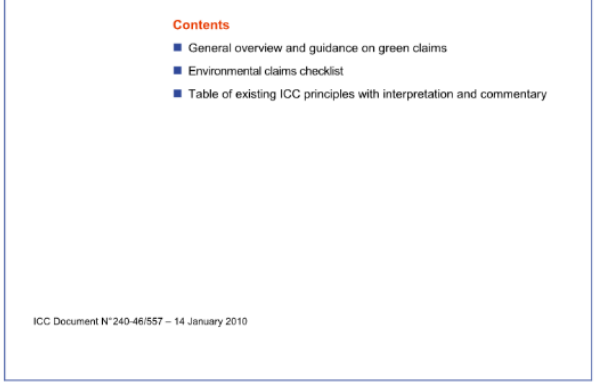

- Environmental claims checkis

Figure 2. ICC Code closely, in particular where moving image ads and still image ads target different audiences which meet them in different places and process them differently. Moving images are usually placed in the advertisement spot of TV channels or on video-sharing websites such as Youtube. In this case we cannot define an audience, and therefore this type of advertisement is usually made simple but noticeable in order to raise awareness. It requires what we will later call heuristic processing. If we want to reach a sophisticated social class we have to find another effective solution: one possible way is to communicate through still images in thematic journals.

Another way in which WWF ads are targeted at different audiences is the complexity of the visual argumentation structure. This also reflects the Framework's recommendation.

On the other hand, the visual argumentation structure can segregate consumers. Not every picture has an argumentative role, but if it does, processing it will be a challenge for

\footnotetext{
${ }^{7}$ If the ice falls, we all fall 05. 06. 2010. http://osocio.org/message/if-the-ice-falls-we-all-fall/ last accessed 11. 12. 2015.

${ }^{8}$ WWF Stop One. Stop Them All. campaign 09. 01. 2014.http://www.commarts.com/exhibit/wwf-stop-onestop-them last accessed 11. 12. 2015.

${ }^{9}$ WWF Campaign by Marcin Budziński 27. 09. 2013. http://www.gutewerbung.net/wwf-campaign-by-marcinbudzinski/ last accessed 11. 12. 2015.

${ }^{10}$ International Chamber of Commerce (ICC), "Policy commissions", http://www.iccwbo.org/about-icc/policycommissions/ last accessed 06.12.2015.

11 International Chamber of Commerce (ICC), "Framework for Responsible Environmental Marketing Communications", p. 7., ICC Document N²40-46/665 2011. July
} 
professional users as well. The Framework adds, "Moreover, even reasonable consumers may have different interpretations of one claim presented in a particular context. Advertising the environmental aspects of a product often requires qualification and additional explanation, not merely the use of buzz words to attract consumers." ${ }^{12}$ This effort to understand visual argumentation is presumed to enhance attitude change through systematic processing.

\section{Systematic and/or heuristic processing}

The Heuristic-Systematic Model of Information Processing is a widely recognised communication model proposed by Shelly Chaiken ${ }^{13}$ that attempts to explain how people receive and process persuasive messages. The model states that individuals can process messages in one of two ways: heuristically or systematically. Heuristic processing uses judgmental rules known as knowledge structures that are learned and stored in memory. These are easily available patterns of inferences or decision procedures which, however, result in right judgments only if certain special conditions are fulfilled. These conditions obtain in the most frequent cases, so these processes usually lead to correct solutions. They are, so to speak, quick-and-dirty rules. Systematic processing, on the other hand, requires conscious effort and is time-consuming, but it is not tied to special conditions. Consequently, it leads to correct judgments even under atypical conditions. ${ }^{14}$

This model ties in with our topic in the following way. Ads directed at a less sophisticated or attentive audience should facilitate heuristic processing whereas those directed at a more sophisticated or more attentive audience should trigger systematic processing.

These two processing methods are very useful when a public service campaign is intended to achieve the maximum effect, as it must be eye-catching, informative and persuasive. I have often found that the first criterion is fully implemented (containing more heuristic elements), while the quantity and quality of the arguments are limited (containing fewer systematic elements). Moreover, typical consumers have to devote more time to understanding the main messages and logical structures of pictures.

Systematic processing involves attempts to thoroughly understand any available information through careful attention, deep thinking, and intensive reasoning. In other words this processing method is comprehensive and analytic. If we see a picture and want to explore the argumentative content, we have to analyse it systematically. ${ }^{15}$

\footnotetext{
12 International Chamber of Commerce (ICC), "Framework for Responsible Environmental Marketing Communications", p. 7., ICC Document N²40-46/665 2011. July

13 Albarracin, D., Johnson, B. T., \& Zanna, M. P., "The handbook of attitudes." 2005.

${ }^{14}$ Paul A. M. Van Lange \& Arie W. Kruglanski \& E. Tory Higgins, "Handbook of Theories of Social Psychology: Volume 1", 2012.

${ }^{15}$ Paul A. M. Van Lange \& Arie W. Kruglanski \& E. Tory Higgins, "Handbook of Theories of Social Psychology: Volume 1", 2012.
} 


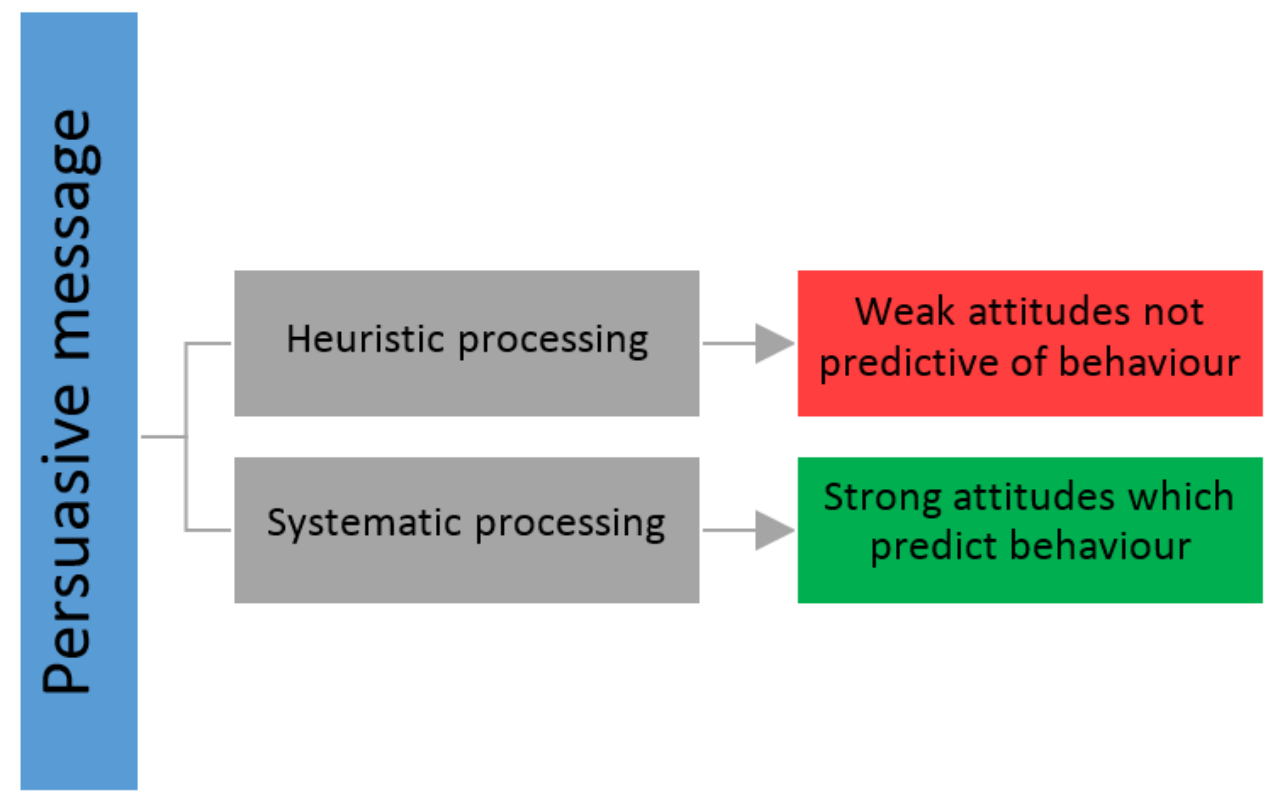

Figure 3. Heuristic vs. Systematic processing

We can state that the way we process information is linked to our attitudes (which means our beliefs and behaviours towards some object) and changes in our attitudes.

Figure $3^{16}$ indicates the types of attitude change that can result from using heuristic and systematic processing. If people are not highly motivated or have low cognitive abilities, they prefer to use the former processing method. Instead of focusing on the argument, they are most likely to appreciate vivid information, social proof, reciprocity, authority and liking. This way of processing information is unlikely to lead to permanent attitude changes. The beliefs and preferences resulting from the messages are temporary and unstable. In contrast, if people are motivated to pay attention to the campaign messages or images, they are also prompted to understand the logic of the arguments as thoroughly as possible. Attitudes emerging from this type of processing tend to be more stable.

\section{Visual arguments and schemes}

To sum up the theoretical background in broad terms, we can observe that the processing and cognition of images are influenced by two types of method, which are conclusively based on visual arguments. We have to put forward what a visual argument is, and why and when we have to recognise it. In this essay, I will not attempt to provide a detailed analysis of the visual argument theory, but I will draw on a few crucial elements.

Before starting to analyse the visual arguments in the WWF ads, let me put to rest any doubts readers may have concerning the very existence of visual arguments. In his Logic, Art and Argument Leo Groarke responds to the usual objections against visual arguments. He makes the following points:

\footnotetext{
${ }^{16}$ Richard J. Crisp, Rhiannon N Turner, “Essential Social Psychology”, (2007.)
} 
1. The arational rhetoric aspect is not unique to visual argument, because it is also an integral part of verbal argumentation.

2.1. Emotion and indefiniteness are usually associated with visual images as opposed to words. However, we can also sufficiently articulate emotions by using sentences.

2.2. It is a misleading generalization that whereas verbal expressions are precise and definite, images are vague and ambiguous. Visual arguments can be sufficiently accurate.

3. While the implicitness of visual arguments is usually highlighted, it has an analogue in implicit or hidden premises and conclusions that accompany many verbal claims.

4. Verbal and, obviously, visual arguments can contain a premise-conclusion structure, which can be investigated by the common standards of argumentative analysis and reasoned persuasion. Argumentation fallacies are also typical of visual argumentation. 17

Here, I have to add that in the WWF poster campaigns I will also examine 'multimodal visual arguments'. This term was introduced by Blair in Probative Norms for Multimodal Visual Arguments for visual arguments which are "actually hybrids or 'multimodal': they will introduce verbal components, but their successful expression depends also on their visual components." 18

When I explore visual premises and their conclusions, I will arrange them into argumentation schemes, abstract structures which capture the common features of similar arguments. They are schemes in the sense that different replacements of the variable they contain yield concrete arguments. ${ }^{19}$ The schemes most extensively used in the selected WWF campaigns are arguments from analogy, reasonings from negative consequences and slippery slope arguments. ${ }^{20}$

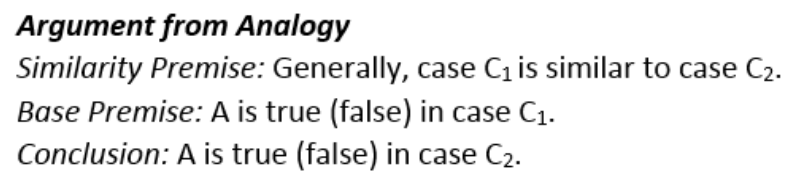

\section{Slippery Slope Argument}

First Step Premise: $\mathrm{A}_{0}$ is up for consideration as a proposal that seems initially like something that should be brought about.

Recursive Premise: Bringing up $\mathrm{A}_{0}$ would plausibly lead (in the given circumstances, as far as we know) to $A_{1}$, which would in turn plausibly lead to $A_{2}$, and so forth, though the sequence $A_{2}, \ldots A_{n}$. Bad Outcome Premise: $A_{n}$ is a horrible (disastrous, bad) outcome.

Conclusion: $\mathrm{A}_{0}$ should not be brought about.

Figure 4. Argumentation schemes

\footnotetext{
${ }^{17}$ Leo Groarke, “Logic, Art and Argument", Informal Logic, vol.18, no. 2 \&3 (1996.) pp. 105-129.

18 J. Anthony Blair, "Probative Norms for Multimodal Visual Arguments", Argumentation (12. 09. 2014)

19 Doug N. Walton and Chris A. Reed, “Argumentation Schemes and Defeasible Inferences" http://cgi.csc.liv.ac.uk/ floriana/CMNA/WaltonReed.pdf last accessed: 12.12.2015.

${ }^{20}$ Macagno, Reed and Walton, "Argumentation Schemes", Cambridge University Press (2008.)
} 


\section{Moving Image}

The most significant characteristic of moving images is their short duration (typically 30 seconds), which means that the heterogeneous audience cannot devote a long time to interpreting the content. Moreover, they usually appear in an advertisement break where several short clips follow each other in a chain, which means that it is difficult to recall them.

On the one hand people prefer watching infotainment advertisements, which are full of remarkable elements and symbols (i.e. dogs, cats, babies, friends, extreme and unique things etc.). On the other hand if people hear/read/watch a narrative story with typical elements like heroes, villains and sensational content - they tend to recall them better. If the content is not sensational, they will not remember it clearly.

WWF's short movie "It all comes back to you" 21 tells a narrative story without narration. In addition, the end of the story is shocking and remarkable. I have found that the "eyecatching" criterion is fully implemented, while the quantity and quality of the argument is limited. See a reconstruction below.

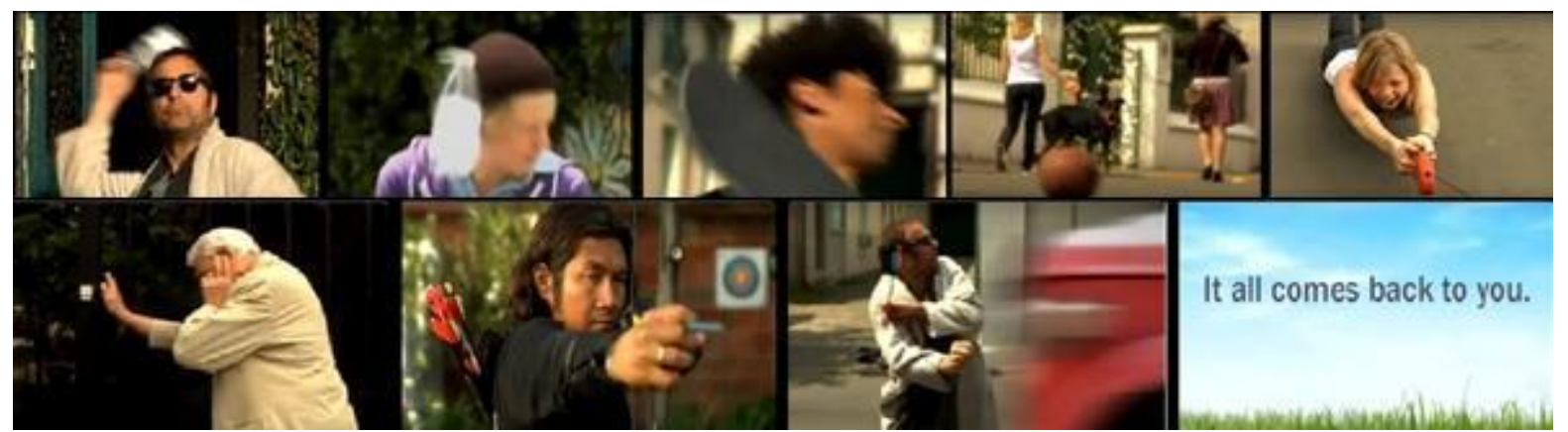

Figure 5. It all comes back to you

\section{Slippery Slope Argument}

First Step Premise:

Recursive Premise:

Bad Outcome Premise:

Conclusion:
Pollution (i.e. throwing away the PET bottle)

Pollution could plausibly lead (in the given circumstances, as far as we know) to the bottle hitting the skateboarder's head, which would in turn plausibly result in his skateboard flying away and hitting the basketball player in the neck, the basketball rolling away, a nearby dog running after the ball while pulling on the leash of its walker, who screams. This will lead to an elderly man's hearing aid whistling. The elderly man will ring the doorbell, disturbing the archer as he shoots and causing him to miss his aim, so the arrow hits the littering man. The littering man, blinded by pain, staggers out on the road where he is run over by a truck.

Being run over by a truck is a horrible outcome.

Pollution should not be brought about, because "it all comes back to you".

\footnotetext{
${ }^{21}$ Youtube: It all comes back to you, https://www.youtube.com/watch?v=pkQx7rqv3Ms last accessed: 15.12.2015.
} 
In this case, the processing method is based on the heuristic approach, but when we analyse its argumentation structure we are using the systematic method. This slippery slope argument invokes a causal chain which leads to the wrong conclusion establishing a misleading generalization.

\section{Still images}

Fields of use for still images are broader, as they can appear in thematic journals, city lights and posters. Moreover, the time frame for processing content is longer, so that viewers have an opportunity to recognize complex argumentation schemes.

In WWF's poster campaign, two different processing methods are integrated with each other. The posters are required to be eye-catching and remarkable, but if they are not built on a strong argumentative structure, the main message will not be effective and will cause contradictory impact.

Slippery slope-type arguments are particularly widespread, because these logical chains are easy to represent well. Normally, these argumentation schemes cannot confirm a conclusion.

However, in the If the trees/ice fall, we all fall posters, the argumentation structure will be valid because these base elements, such as ice and trees, are symbolised as a universal category. Thus the pictures convincingly demonstrate that the result is not an inductive syllogism, but something we would rather refer to as a deductive syllogism.

In line with this categorisation, see the pictures and their reconstruction below:

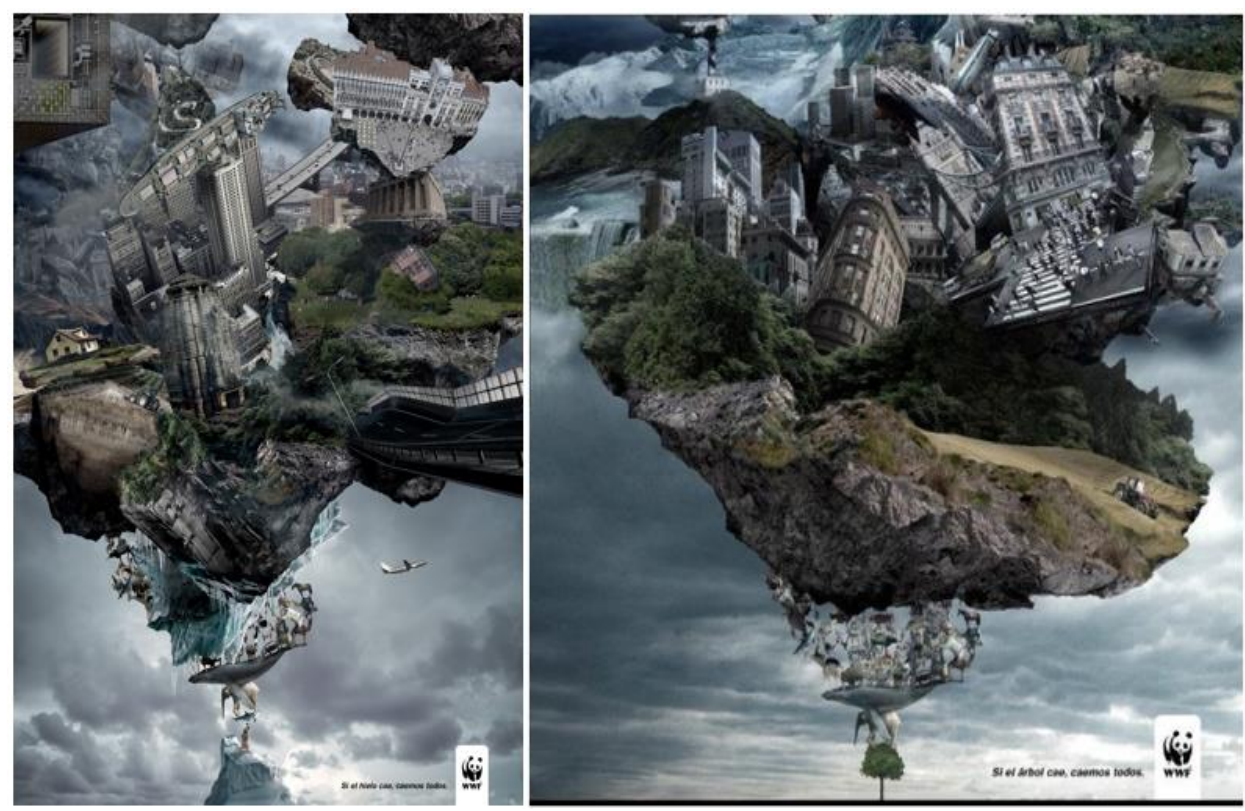

Figure 6. If the trees/ice fall, we all fall 


\section{Slippery Slope Argument I.}

First Step Premise: We reinforce the greenhouse effect.

Recursive Premise: If we reinforce the greenhouse effect, the ice will melt.

If the ice melts, it will destroy the ecosystem.

If the ecosystem is destroyed, humanity will become extinct.

Bad Outcome Premise: The extinction of humanity is avoidable.

Conclusion: It is necessary to avoid reinforcing the greenhouse effect.

\section{Slippery Slope Argument II.}

First Step Premise: We destroy our forests.

Recursive Premise: If we destroy our forests, we will upset the balance of the ecosystem.

If we upset the balance of the ecosystem, humanity will become extinct.

Bad Outcome Premise: The extinction of humanity is avoidable.

Conclusion:

It is necessary to avoid deforestation.

I will now present an invalid slippery slope argument, where the conclusion is ambiguous. In this picture, we can recognise multimodal visual arguments, because the written part of images ("Stop one. Stop them all.") is one of the main premises. This campaign is against illegal hunting/fishing and illustrates on whom this process is built.
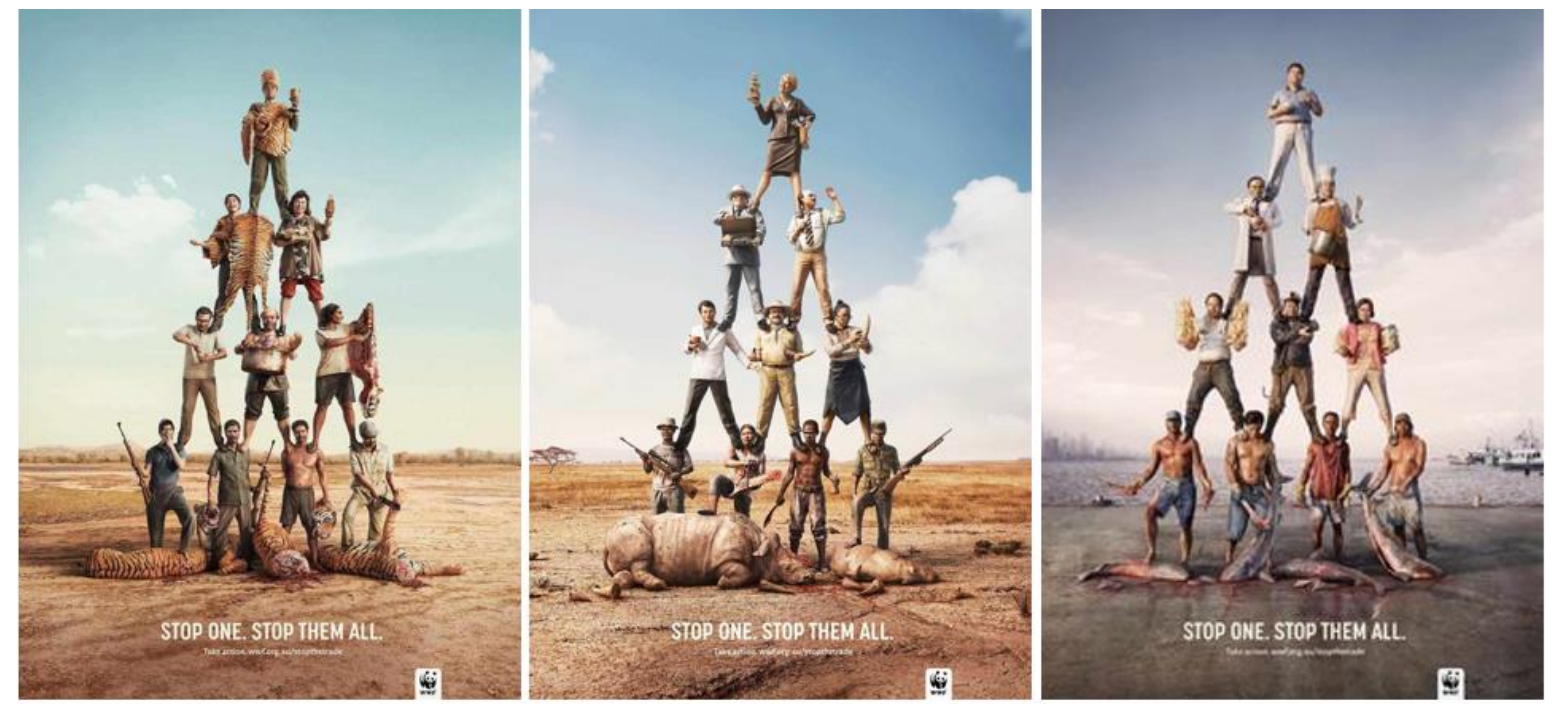

Figure 7. Stop one. Stop them all. 


\section{Slippery Slope Argument}

First Step Premise: $\quad$ You stop the end-user.

Recursive Premise: If you stop the end-user, you will not need the reseller.

If you don't need a reseller, the animal's carcass will not need processing.

If the animal's carcass doesn't need processing, you will not need a hunter.

Bad Outcome Premise: Because of the end-user, hunting has got horrible consequences.

Conclusion:

To sum up, stopping the end-user will stop the whole process.

This is a misleading visual representation, because it does not support the argument. We can ask the question: which member of the pyramid is the only guilty one; whose actions are wrong? The pyramid will not collapse if we take out the end user. My suggestion would be to turn the pyramid upside down, or take out one of the hunters or middle men.

I have to acknowledge that the stages of animal carcass processing are visually well represented in this campaign. Moreover, a pyramid structure suggests a hierarchical relationship, but if WWF intends to achieve attitude change, it needs to focus on the crucial argumentative content.

This is a case study of a typical hybrid visual argumentation: if the viewer does not recognise the subtitle, this picture cannot convey WWF's mission.

The poster series Stop one. Stop them all contains fewer remarkable elements, but another campaign, Bycie pamiqtkq boli [Being a trophy hurts], contains more noticeable, shocking representations. It is a double-edged communication strategy, because one part of the viewers will be struck by them, but will not devote time to understanding their message. The other part of the audience will be shocked too, but they will never forget the images: they will make an effort to recognise the content and may well change their attitudes.

Arguments from analogy can be very effective if viewers can establish a strong connection between the two objects being compared. In the next case, the brutality of people represented in the pictures is inverted in every field of life.

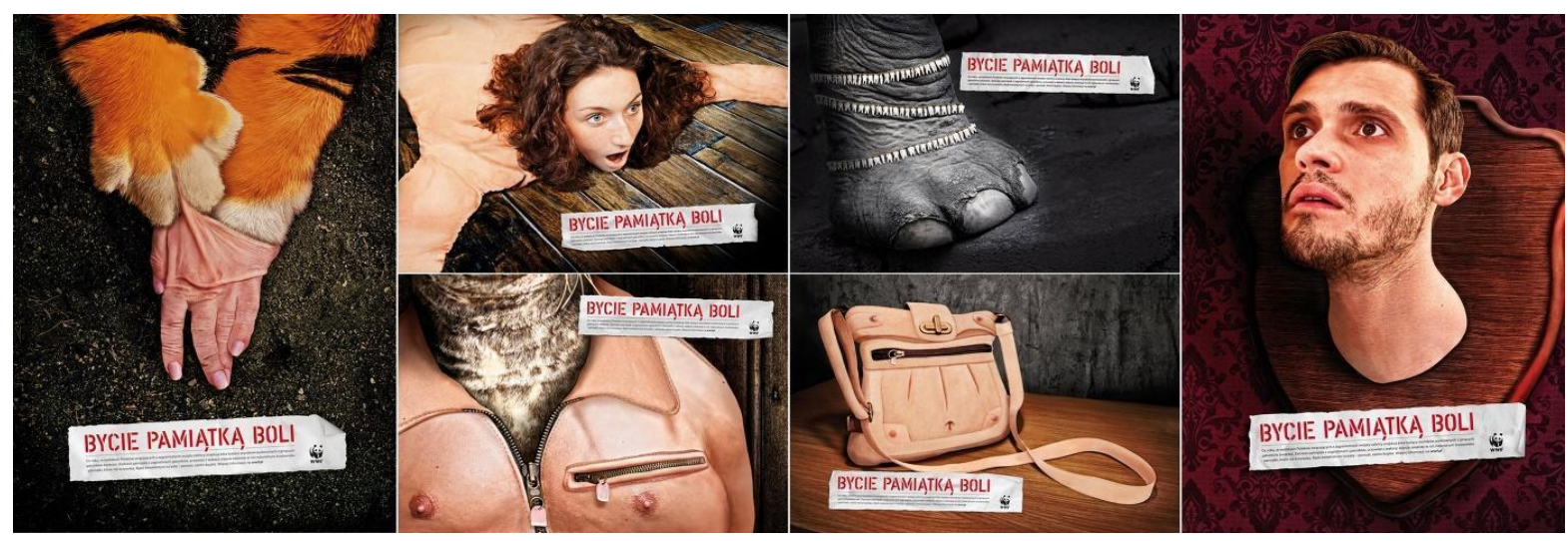

Figure 8. Being a trophy hurts 


\section{Argument from Analogy}

Implicit Base Premise:

People wear/use gloves/jackets/bags/necklaces/rugs/trophies, which are made from animals.

Similarity Premise:

In order to use these accessories they have to butcher animals.
Visual Base Premise:

Animals are wearing/using gloves/a jacket/a bag/a necklace/a rug/a trophy, which are made from humans.

Conclusion:

In order to use these accessories they have to butcher humans.

To understand a visual argumentation it is necessary to presuppose an implicit base and visual premises. This identification certainly demonstrates that this is a systematic cognitive process. The visual argument is complex, because the argument from analogy is only a starting point, which is followed by the type of reasoning from negative consequence.

\section{Reasoning from Negative Consequences}

Premise 1:

Premise 2:

Conclusion:
If people treat animals in brutal and cruel ways to satisfy their own selfish goals, it will bring about serious consequences.

People will cause animals suffering: that is a serious consequence.

Therefore, we should not be cruel to animals.

\section{Conclusion}

In my paper I analysed World Wildlife Fund (WWF) public service campaigns - It all comes back to you, If the ice/trees fall, we all fall, Stop one. Stop them all and Bycie pamiqtkq boli focussing on the visual elements of understanding.

I argued that visual argumentation can influence the processing and cognition of images. I asserted that moving images contain fewer argumentative elements as they prefer to operate with heuristic visual representations. I also confirmed that the components of still images are more argumentative in order to achieve attitude change. WWF has to conform to the ICC Code, which states that a communication campaign message must suit a variously qualified audience. 
To sum up my research results, I will provide some elements of comparison in the following chart:

\section{Moving image}

\section{Still image}

\section{Similarities}

Both contain heuristic elements in order to be eye-catching.

Both can be analysed using the argumentation theory.

Short time to recognise content.

Long time to recognise content.

Does not typically contain complex

Differences argumentative structures.

Is a good example of how the heuristic processing method works.
Typically contains complex argumentative structures.

Is a good example of how the systematic processing method works.

Thus, I have shown that there is a connection between argumentation structures and the processing of images (still and moving).

\section{Acknowledgements}

Special thanks go to Gábor Forrai PhD, János Tanács PhD, István Danka PhD and Dénes Tamás and all of the colleagues at the Department of Philosophy and History of Science, Budapest University of Technology and Economics, who helped me with their advice during my research. 